\title{
VOLUME MINIMIZATION AND ESTIMATES FOR CERTAIN ISOTROPIC SUBMANIFOLDS IN COMPLEX PROJECTIVE SPACES*
}

\author{
EDWARD GOLDSTEIN ${ }^{\dagger}$
}

\begin{abstract}
In this note we show the following result using the integral-geometric formula of R. Howard: Consider the totally geodesic $\mathbb{R} P^{2 m}$ in $\mathbb{C} P^{n}$. Then it minimizes volume among the isotropic submanifolds in the same $\mathbb{Z} / 2$ homology class in $\mathbb{C} P^{n}$ (but not among all submanifolds in this $\mathbb{Z} / 2$ homology class). Also the totally geodesic $\mathbb{R} P^{2 m-1}$ minimizes volume in its Hamiltonian deformation class in $\mathbb{C} P^{n}$. As a corollary we'll give estimates for volumes of Lagrangian submanifolds in complete intersections in $\mathbb{C} P^{n}$.
\end{abstract}

Key words. Isotropic submanifolds, Lagrangian submanifolds, volume minimization

AMS subject classifications. $53 \mathrm{XX}$

1. Introduction. On a Kähler $n$-fold $M$ there is a class of isotropic submanifolds. Those are submanifolds of $M$ on which the Kähler form $\omega$ of $M$ vanishes. The maximal dimension of such a submanifold is $n$ (the middle dimension) in which case it is called Lagrangian.

In this papers we'll exhibit global volume-minimizing properties among isotropic competitors for certain submanifolds of the complex projective space. In general global volume-minimizing properties of minimal/Hamiltonian stationary Lagrangian/isotropic submanifolds in Kähler (particularly Kähler-Einstein) manifolds are still poorly understood. In dimesion 2 there is a result of Schoen-Wolfson [ScW] (extended to isotropic case by Qiu in [Qiu]) which shows existence of Lagrangian cycles minimizing area among Lagrangians in a given homology class. Still it is not clear whether a given minimal Lagrangian has any global volume-minimizing properties. The only instance where we have a clear cut answer to global volume-minimizing problem is Special Lagrangian submanifolds which are homologically volume-mimizing in Calabi-Yau manifolds [HaL]. In Kähler-Einstein manifolds of negative scalar curvature, besides geodesics on Riemann surfaces of negative curvature, we have some examples [Lee] of minimal Lagrangian submanifolds which are homotopically volumeminimizing. The author has a program for studying homotopy volume-minimizing properties for Lagrangians in Kähler-Einstein manifolds of negative scalar curvature [Gold1], but so far there are no satisfactory results.

In positive curvature case there is a result of Givental-Kleiner-Oh which states that the canonical totally geodesic $\mathbb{R} P^{n}$ in $\mathbb{C} P^{n}$ minimizes volume in its Hamiltonian deformation class, [Giv]. The proof uses integral geometry and Floer homology to study intersections for Hamiltonian deformations of $\mathbb{R} P^{n}$. Those arguments can be generalized to products of Lagrangians in a product of symmetric Kähler manifolds, [IOS]. There is a related conjecture due to Oh that the Clifford torus minimizes volume in its Hamiltonian deformation class in $\mathbb{C} P^{n},[\mathrm{Oh}]$. Some progress towards this was obtained in [Gold2]. Also general lower bounds for volumes of Lagrangians in a given Hamiltonian deformation class in $\mathbb{C}^{n}$ were obtained in [Vit].

In this note we extend and improve the result of Givental-Kleiner-Oh to isotropic totally geodesic $\mathbb{R} P^{k}$ sitting canonically in $\mathbb{C} P^{n}$. Our main result is the following

\footnotetext{
*Received August 3, 2004; accepted for publication December 16, 2004.

$\dagger$ School of Mathematics, Institute for Advanced Study, 1 Einstein Drive, Princeton, New Jersey, 08540, USA (egold@ias.edu).
} 
theorem:

THEOREM 1. Consider the totally geodesic $\mathbb{R} P^{2 m}$ in $\mathbb{C} P^{n}$. Then it minimizes volume among the isotropic submanifolds in the same $\mathbb{Z} / 2$ homology class in $\mathbb{C} P^{n}$ (but not among all submanifolds in this $\mathbb{Z} / 2$ homology class). Also consider the totally geodesic $\mathbb{R} P^{2 m-1}$ in $\mathbb{C} P^{n}$. Then it minimizes volume in its Hamiltonian deformation class.

A corollary of this is:

COROLlary 1. Let $f_{1}, \ldots, f_{k}$ be real homogeneous polynomials of odd degree in $n+1$ variables with $2 m+k=n$. Let $N$ be the zero locus of $f_{i}$ in $\mathbb{C} P^{n}$ and $L$ be their real locus. Then $\operatorname{vol}(L) \leq \Pi \operatorname{deg}\left(f_{i}\right) \operatorname{vol}\left(\mathbb{R} P^{2 m}\right)$ and if $L^{\prime}$ is a Lagrangian submanifold of $N$ homologous mod 2 to $L$ in $N$ then $\operatorname{vol}\left(L^{\prime}\right) \geq \operatorname{vol}\left(\mathbb{R} P^{2 m}\right)$.

Acknowledgement. The author would like to thank Aleks, Olga and Nelly Neimark for their hospitality during his stay in Princeton, NJ.

2. A formula from integral geometry. In this section we establish a formula from integral geometry for volumes of isotropic submanifolds of $\mathbb{C} P^{n}$ following the exposition in R. Howard [How].

In our case the group $S U(n+1)$ acts on $\mathbb{C} P^{n}$ with a stabilizer $K \simeq U(n)$. Thus we view $\mathbb{C} P^{n}=S U(n+1) / K$ and the Fubini-Study metric is induced from the bi-invariant metric on $S U(n+1)$. Let $P^{2 m}$ be an isotropic submanifold of $\mathbb{C} P^{n}$ of dimension $2 m$ and let $Q$ be a linear $\mathbb{C} P^{n-m} \subset \mathbb{C} P^{n}$. For a point $p \in P$ and $q \in Q$ we define an angle $\sigma(p, q)$ between the tangent planes $T_{p} P$ and $T_{q} Q$ as follows: First we choose some elements $g$ and $h$ in $S U(n+1)$ which move $p$ and $q$ respectively to the same point $r \in \mathbb{C} P^{n}$. Now the tangent planes $g_{*} T_{p} P$ and $h_{*} T_{q} Q$ are in the same tangent space $T_{r} \mathbb{C} P^{n}$ and we can define an angle between them as follows: take an orthonormal basis $u_{1} \ldots u_{2 m}$ for $g_{*} T_{p} P$ and an orthonormal basis $v_{1} \ldots v_{2 n-2 m}$ for $h_{*} T_{q} Q$ and define

$$
\sigma\left(g_{*} T_{p} P, h_{*} T_{q} Q\right)=\left|u_{1} \wedge \ldots \wedge v_{2 n-2 m}\right| .
$$

The later quantity $\sigma\left(g_{*} T_{p} P, h_{*} T_{q} Q\right)$ depends on the choices $g$ and $h$ we made. To mend this we'll need to average this out by the stabilizer group $K$ of the point $r$. Thus we define:

$$
\sigma(p, q)=\int_{K} \sigma\left(g_{*} T_{p} P, k_{*} h_{*} T_{q} Q\right) d k
$$

Since $S U(n+1)$ acts transitively on the Grassmanian of isotropic planes and the complex planes in $\mathbb{C} P^{n}$ we conclude that this angle is a constant depending just on $m$ and $n$ :

$$
\sigma(p, q)=C_{m, n}
$$

There is a following general formula due to R. Howard [How]:

$$
\int_{S U(n+1)} \#(P \bigcap g Q) d g=\int_{P \times Q} \sigma(p, q) d p d q=C_{m, n} \operatorname{vol}(P) \operatorname{vol}(Q) .
$$

Here \# $(P \cap g Q)$ is the number of intersection points of $P$ with $g Q$, which is finite for a generic $g \in S U(n+1)$. To use the formula we need to have some control over the intersection pattern of $P$ and $g Q$. We have the following lemma: 
LEMma 1. Let $P$ be the totally geodesic $\mathbb{R} P^{2 m} \subset \mathbb{C} P^{n}$, let $Q=\mathbb{C} P^{n-m} \subset \mathbb{C} P^{n}$. Let $g \in S U(n+1)$ s.t. $P$ and $g Q$ intersect transversally. Then $\#(P \cap g Q)=1$. Also let $f_{1}, \ldots, f_{k}$ be real homogeneous polynomials in $n+1$ variables with $2 m+k=n$ and let $P^{\prime}$ be their real locus. If $P^{\prime}$ is transversal to $g Q$ then $\#\left(P^{\prime} \cap g Q\right) \leq \Pi \operatorname{deg}\left(f_{i}\right)$.

Proof. For the first claim we have $g Q$ is given by an $(n-m+1)$-plane $H \subset \mathbb{C}^{n+1}$ and hence it is a zero locus of $m$ linear equations on $\mathbb{C}^{n+1}$. Hence $(P \cap g Q)$ is cut out by $2 m$ linear equations in $\mathbb{R} P^{2 m}$.

For the second claim we note that as before $g Q \bigcap \mathbb{R} P^{n}$ is the zero locus of $2 m$ linear polymonials $h_{1}, \ldots, h_{2 m}$ on $\mathbb{R} P^{n}$. Moreover $P^{\prime}$ is a zero locus of $f_{1}, \ldots, f_{n-2 m}$ on $\mathbb{R} P^{n}$. For generic $g \in S U(n+1)$ we'll have that $g Q$ and $P^{\prime}$ intersect transversally in $\mathbb{R} P^{n}$. By Bezout's theorem (see $[\mathrm{GH}]$, p. 670) the common zero locus of $h_{1}, \ldots, h_{2 m}$ and $f_{1}, \ldots, f_{n-2 m}$ is $\mathbb{C} P^{n}$ is $\Pi \operatorname{deg}\left(f_{i}\right)$ points. Now $P^{\prime} \cap g Q$ is a part of this locus, hence $\#\left(P^{\prime} \cap g Q\right) \leq \Pi \operatorname{deg}\left(f_{i}\right)$.

3. Proof of the volume minimization. Now we can prove the result stated in the Introduction:

TheOREM 1. Consider the totally geodesic $\mathbb{R} P^{2 m}$ in $\mathbb{C} P^{n}$. Then it minimizes volume among the isotropic submanifolds in the same $\mathbb{Z} / 2$ homology class in $\mathbb{C P}^{n}$ (but not among all submanifolds in this $\mathbb{Z} / 2$ homology class). Also consider the totally geodesic $\mathbb{R} P^{2 m-1}$ in $\mathbb{C} P^{n}$. Then it minimizes volume in its Hamiltonian deformation class.

Proof. Let $P$ be an isotropic submanifold homologous to $\mathbb{R} P^{2 m} \bmod 2$ and let $Q=\mathbb{C} P^{n-m}$. By Lemma 1 the intersection number mod 2 of $P$ and $g Q$ is 1 . Hence the formula in the previous section tells that

$$
C_{m, n} \operatorname{vol}(P) \operatorname{vol}(Q)=\int_{S U(n+1)} \#(P \bigcap g Q) d g \geq \operatorname{vol}(S U(n+1))
$$

and

$$
C_{m, n} \operatorname{vol}\left(\mathbb{R} P^{2 m}\right) \operatorname{vol}(Q)=\int_{S U(n+1)} \#\left(\mathbb{R} P^{2 m} \bigcap g Q\right) d g=\operatorname{vol}(S U(n+1))
$$

and this proves the first part. We also note that that $\mathbb{C} P^{1}$ is homologous to $\mathbb{R} P^{2} \bmod$ 2 in $\mathbb{C} P^{n}$ but

$$
\operatorname{vol}\left(\mathbb{C} P^{1}\right)<\operatorname{vol}\left(\mathbb{R} P^{2}\right) .
$$

The second assertion will follow from the first one. Consider $\mathbb{C}^{n+1}$ and a unit sphere $S^{2 n+1} \subset \mathbb{C}^{n+1}$. We have a natural circle action on $S^{2 n+1}$ (multiplication by unit complex numbers). Let the vector field $u$ be the generator of this action. We have a 1-form $\alpha$ on $S^{2 n+1}$,

$$
\alpha(v)=u \cdot v
$$

Also $d \alpha=2 \omega$ where $\omega$ is the Kähler form of $\mathbb{C}^{n+1}$. The kernel of $\alpha$ is the horizontal distribution. We have a Hopf map $\rho: S^{2 n+1} \mapsto \mathbb{C} P^{n}$. We have $\mathbb{R} P^{2 m-1} \subset \mathbb{C} P^{n}$ and $S^{2 m-1} \subset S^{2 n+1}$ which is a horizontal double cover of $\mathbb{R} P^{2 m-1}$.

Let $f$ be a (time-dependent) Hamiltonian function on $\mathbb{C} P^{n}$. Then we can lift it to a Hamiltonian function on $\mathbb{C}^{n+1}-(0)$ and its Hamiltonian vector field $H_{f}$ is horizontal on $S^{2 n+1}$. Consider now the vector field

$$
w=-2 f \cdot u+H_{f}
$$


The vector field $w$ is $S^{1}$-invariant. We also have:

Proposition 1. The Lie derivative $L_{w} \alpha=0$.

Proof. We have

$$
L_{w} \alpha=d\left(i_{w} \alpha\right)+i_{w} d \alpha=-2 d f+2 d f .
$$

Let now $\Phi_{t}$ be the time $t$ flow of $w$ on $S^{2 n+1}$ and let $\Xi_{t}$ be the Hamiltonian flow of $f$ on $\mathbb{C} P^{n}$. Then $\Phi_{t}\left(S^{2 m-1}\right)$ is horizontal and isotropic and it is a double cover of $\Xi_{t}\left(\mathbb{R} P^{2 m-1}\right)$. Hence

$$
\operatorname{vol}\left(\Phi_{t}\left(S^{2 m-1}\right)\right)=2 \operatorname{vol}\left(\Xi_{t}\left(\mathbb{R} P^{2 m-1}\right)\right) .
$$

Let $S_{t}=\Phi_{t}\left(S^{2 m-1}\right)$. We build a suspension $\Sigma S_{t}$ of $S_{t}$ in $S^{2 n+3} \subset \mathbb{C}^{n+2}$,

$$
\Sigma S_{t}=\left((\sin \theta \cdot x, \cos \theta) \in \mathbb{C}^{n+2}=\mathbb{C}^{n+1} \oplus \mathbb{C} \mid 0 \leq \theta \leq \pi, x \in S_{t}\right) .
$$

One immediately verifies that $\Sigma S_{t}$ is horizontal and it is a double cover of an isotropic submanifold $L_{t}$ (with a conical singularity) of $\mathbb{C} P^{n+1}$ with $L_{0}=\mathbb{R} P^{2 m}$. Also one readily checks that

$$
\operatorname{vol}\left(\Sigma S_{t}\right)=\operatorname{vol}\left(S_{t}\right) \cdot \int_{\theta=0}^{\pi} \sin ^{2 m-1} \theta d \theta
$$

Hence

$$
2 \operatorname{vol}\left(L_{t}\right)=\operatorname{vol}\left(\Sigma S_{t}\right)=2 \operatorname{vol}\left(\Xi_{t}\left(\mathbb{R} P^{2 m-1}\right)\right) \cdot \int_{\theta=0}^{\pi} \sin ^{2 m-1} \theta d \theta .
$$

Now the first part of our theorem implies that $\operatorname{vol}\left(L_{t}\right) \geq \operatorname{vol}\left(L_{0}\right)$. Hence we conclude that $\operatorname{vol}\left(\Xi_{t}\left(\mathbb{R} P^{2 m-1}\right)\right) \geq \operatorname{vol}\left(\mathbb{R} P^{2 m-1}\right)$.

REMARK. One notes from the proof that for $\mathbb{R} P^{2 m-1}$ it would be suffient to use exact deformations by isotropic immersions of $\mathbb{R} P^{2 m-1}$. A family $L_{t}$ of isotropic immersions of $\mathbb{R} P^{2 m-1}$ is called exact if the 1 -form $i_{v} \omega$ is exact when restricted to each element of the family. Here $v$ is the deformation vector field and $\omega$ is the symplectic form. Thus embeddedness is not important for the conclusion of the theorem. The theorem has the following corollary:

Corollary 1. Let $f_{1}, \ldots, f_{k}$ be real homogeneous polynomials of odd degree in $n+1$ variables with $2 m+k=n$. Let $N$ be the zero locus of $f_{i}$ in $\mathbb{C} P^{n}$ and $L$ be their real locus. Then $\operatorname{vol}(L) \leq \Pi \operatorname{deg}\left(f_{i}\right) \operatorname{vol}\left(\mathbb{R} P^{2 m}\right)$ and if $L^{\prime}$ is a Lagrangian submanifold of $N$ homologous mod 2 to $L$ in $N$ then $\operatorname{vol}\left(L^{\prime}\right) \geq \operatorname{vol}\left(\mathbb{R} P^{2 m}\right)$.

Proof. We note that $N$ is a complex $2 m$-fold and $L$ is its Lagrangian submanifold. Since the degrees of $f_{i}$ are odd, we have by adjunction formula that $L$ and $\mathbb{R} P^{2 m}$ represent the same homology class in $H_{2 m}\left(\mathbb{R} P^{n}, \mathbb{Z} / 2\right)$. Let $Q$ be a linear $\mathbb{C} P^{n-m}$ in $\mathbb{C} P^{n}$ and $g \in S U(n+1)$. The intersection munber mod 2 of $g Q$ with $L^{\prime}$ is 1 . We have that

$$
\begin{gathered}
C_{m, n} \operatorname{vol}\left(\mathbb{R} P^{2 m}\right) \operatorname{vol}(Q)=\int_{S U(n+1)} 1 d g \\
C_{m, n} \operatorname{vol}\left(L^{\prime}\right) \operatorname{vol}(Q)=\int_{S U(n+1)} \#\left(L^{\prime} \bigcap g Q\right) d g .
\end{gathered}
$$


Also using Lemma 1:

$$
C_{m, n} \operatorname{vol}(L) \operatorname{vol}(Q)=\int_{S U(n+1)} \#(L \bigcap g Q) d g \leq \Pi \operatorname{deg}\left(f_{i}\right) \operatorname{vol}(S U(n+1))
$$

and our claims follow.

\section{REFERENCES}

[Giv] A. Givental, The Nonlinear Maslov index, London Mathematical Society Lecture Note Series, 15 (1990), pp. 35-43.

[Gold1] EdWARD GoldSTEIN, Strict volume-minimizing properties for Lagrangian submanifolds in complex manifolds with positive canonical bundle, math.DG/0301191.

[Gold2] Edward Goldstein, Some estimates related to Oh's conjecture for the Clifford tori in $\mathbb{C} P^{n}$, math.DG/0311460.

[GH] P. Griffiths and J. Harris, Principles of Algebraic geometry, Wiley and Sons, 1978.

[HaL] R. Harvey and H. B. Lawson, Calibrated Geometries, Acta Math., 148 (1982), pp. 47157.

[How] Howard, RALPh, The kinematic formula in Riemannian homogeneous spaces, Mem. Amer. Math. Soc., 106 (1993), no. 509, vi+69 pp.

[IOS] Hiroshi IriYen, Hajime Ono, TAKashi SAKai, Integral Geometry and Hamiltonian volume minimizing property of a totally geodesic Lagrangian torus in $S^{2} \times S^{2}$, Proc. Japan Acad. Ser. A Math. Sci., 79:10 (2003), pp. 167-170.

[Lee] Y.-I. LEE, Lagrangian minimal surfaces in Kähler-Einstein surfaces of negative scalar curvature, Comm. Anal. Geom., 2:4 (1994), pp. 579-592.

[Oh] Y.-G. Он, Mean curvature vector and symplectic topology of Lagrangian submanifolds in Einstein-Kähler manifolds, Math. Z., 216 (1994), pp. 471-482.

[Qiu] QIu, WeIYANG, Interior regularity of solutions to the isotropically constrained Plateau problem, Comm. Anal. Geom., 11:5 (2003), pp. 945-986.

[ScW] Schoen, R. And Wolfson, J., Minimizing area among Lagrangian surfaces: the mapping problem, J. Differential Geom., 58:1 (2001), pp. 1-86.

[Vit] C. Viterbo, Metric and isoperimetric problems in symplectic geometry, J. Amer. Math. Soc., 13:2 (2000), pp. 411-431. 
E. GOLDSTEIN 